\title{
WEAKLY UU RINGS
}

\author{
By
}

\author{
Peter V. DANCheV
}

\begin{abstract}
We introduce and give a comprehensive study of weakly $U U$ rings, calling them $W U U$ rings. This notion is a natural generalization of the so-called UU rings, defined by Calugareanu (Carpath. J. Math., 2015) and investigated in details by DanchevLam (Publicat. Math. Debrecen, 2016). It also demarcates the strength of recent results about these kind of rings by giving a strong barrier between some of their crucial properties.
\end{abstract}

\section{Introduction and Background}

Throughout the present paper all rings considered, unless otherwise noted, shall be assumed to be associative, containing identity element. As usual, $U(R)$ denotes the set of all invertible elements of $R, I d(R)$ the set of all idempotents of $R$ and $\operatorname{Nil}(R)$ the set of all nilpotents of $R$. When $R$ is commutative, $\operatorname{Nil}(R)=N(R)$ is just the nil-radical of $R$. Likewise, $J(R)$ stands the Jacobson radical of $R$. All other notions and notations, not explicitly defined herein, will be the standard ones which may be found in [1] and [9], respectively. For instance, referring to [10], a ring $R$ is called clean if each element is the sum of a unit and an idempotent. Likewise, a ring $R$ is said to be exchange if, for any $a \in R$, there exists an idempotent $e \in a R$ such that $1-e \in(1-a) R$. Clean rings are always exchange, while the converse is false; however for abelian rings these two concepts are tantamount.

In [3], a ring $R$ is called $U U$ if all units are unipotents, that is, $U(R)=$ $\operatorname{Nil}(R)+1$, i.e., each unit can be presented as $q+1$, where $q \in \operatorname{Nil}(R)$. This is obviously tantamount to the equality $U(R)=\operatorname{Nil}(R)-1$, that is, each unit can be presented as $q-1$, where $q \in \operatorname{Nil}(R)$. Although this could be showed directly,

2010 Mathematics Subject Classification: 16D50, 16D60, 16 D80.

Key words and phrases: Clean rings, UU rings, WUU rings, Units, Nilpotents.

Received December 16, 2015.

Revised May 16, 2016. 
notice also that the element $2 \in R$ is always a central nilpotent in a $U U$ ring $R$, and thus $q+1=(q+2)-1$ with $q+2 \in \operatorname{Nil}(R)$. So, it is natural to ask when any unit can be represented as either $q+1$ or $q-1$, where $q \in \operatorname{Nil}(R)$. In what follows we call such units weak unipotents, and if all units are so the rings are called $W U U$ rings.

It was obtained in [7, Theorem $\mathrm{A}]$ that a $\mathrm{UU}$ ring $R$ is precisely such a ring $R$ that $\operatorname{char}(R)$ is a power of 2 and $U(R)$ is a 2-group. This characterization helps to be obtained there in an easier form some principal well-known classical results in that direction of ring theory.

The objective of this article is to generalize considerably almost all results in [7] to this new point of view. In fact, we shall somewhat establish the complete description of clean WUU rings (Corollary 2.14) as well as the complete description of weakly nil-clean rings (Theorem 2.17) and weakly nil-regular rings (Proposition 2.20). Moreover, a characterization of clean and weakly nil-clean elements in abelian WUU rings with $6 \in \operatorname{Nil}(R)$ is given too (Theorem 2.21).

The work is organized as follows: Here, in the first section, we stated the main instruments and principal known results needed for a good presentation. In the second section we deal with WUU rings and establish their crucial properties. We close with a third section, containing a series of left-open challenging problems.

\section{WUU Rings}

The following new concept is our starting point of view.

Definition 2.1. A ring $R$ is called weakly $U U$, and abbreviated as $W U U$, if $U(R)=\operatorname{Nil}(R) \pm 1$. This is equivalent to the condition that every unit can be presented as either $n+1$ or $n-1$, where $n \in \operatorname{Nil}(R)$.

The following are non-trivial examples of WUU rings, thereby showing that there is a plenty of them:

ExAmple 2.2. (a) A ring $R$ is said to be in [8], and generally in [2], weakly nil-clean if each $r \in R$ can be written as $r=n+e$ or $r=n-e$, where $n \in \operatorname{Nil}(R)$ and $e \in I d(R)$. If $n e=e n$, then the weakly nil-clean ring is said to have the strong property - see [6]. By [8, Corollary 1.16], commutative weakly nil-clean rings are WUU rings. In particular, $\mathbf{Z}_{3}$ is a WUU ring. 
(b) Any UU ring is WUU. In particular, by [7], $\mathbf{Z}_{2}$ is a $\mathrm{UU}$ ring and so a WUU ring.

(c) The ring $\mathbf{T}_{n}\left(\mathbf{Z}_{2}\right)$ of all upper triangular $n \times n$ matrices being UU is also a WUU ring, whereas the ring $\mathbf{T}_{n}\left(\mathbf{Z}_{3}\right)$ is not (compare also with Corollary 2.28 stated below).

(d) The ring $\mathbf{Z}$ of all integers is WUU but not UU because $U(\mathbf{Z})=\{-1,1\}$. However, it is not an exchange ring.

We can now strengthen Example 2.2(a) a little bit. Recall that a ring $R$ is abelian provided all its idempotents are central.

Proposition 2.3. Abelian weakly nil-clean rings are $W U U$.

Proof. By [2, Theorem 12] such a ring $R$ is precisely the ring $R$ with nil $J(R)$ and $R / J(R)$ is isomorphic to either a boolean ring, or to $\mathbf{Z}_{3}$, or to the product of two such rings. Thus, either $U(R / J(R)) \cong\{1\}$ or $U(R / J(R)) \cong$ $\{-1,1\}$. Furthermore, because of the inclusion $J(R) \subseteq N i l(R)$ and the validity of the isomorphism

$$
U(R) /(1+J(R)) \cong U(R / J(R)),
$$

we deduce that $U(R)= \pm 1+\operatorname{Nil}(R)$, as wanted.

Although homomorphic images of units, idempotents and nilpotents are again units, idempotents and nilpotents, respectively, it follows in general that even an epimorphic image of a WUU ring need not be WUU as well. For instance, as showed in Example $2.2(\mathrm{~d})$, the $\operatorname{ring} \mathbf{Z}$ is $\mathrm{WUU}$, while $\mathbf{Z}_{p} \cong \mathbf{Z} /(p)$ is not so unless $p=2$ or $p=3$ (compare with Corollary 2.7 below). However, the following is valid:

Lemma 2.4. Suppose $R$ is a ring with $I \triangleleft J(R)$. Then $R / I$ is $W U U$, provided that $R$ is WUU.

Proof. We claim that the group map $U(R) \rightarrow U(R / I)$, induced by the canonical ring epimorphism $R \rightarrow R / I$, is a surjective group homomorphism. In fact, that it is a homomorphism of groups follows easily. To treat the surjection, if $u v+I=(u+I)(v+I)=(v+I)(u+I)=v u+I=1+I$ for some $u, v \in R$, then $u v \in 1+I \subseteq 1+J(R) \subseteq U(R)$ as well as $v u \in 1+I \subseteq 1+J(R) \subseteq U(R)$. 
These two inclusions manifestly show that $u \in U(R)$, as claimed. Furthermore, by what we have just shown, if $w=u+I \in U(R / I)$, then $u \in U(R)= \pm 1+\operatorname{Nil}(R)$, so that $u+I= \pm(1+I)+(q+I)$ for some $q \in \operatorname{Nil}(R)$ whence $w \in \pm(1+I)+$ $\operatorname{Nil}(R / I)$, as needed.

Proposition 2.5. Suppose $I$ is a nil-ideal of a ring $R$. Then $R$ is $W U U$ if and only if $R / I$ is $W U U$.

Proof. Since $I \subseteq J(R)$ always, in view of Lemma 2.4, we need to prove only the "if" part. To that goal, assume that $r \in U(R)$. Hence $r+I \in U(R / I)=$ $\pm(1+I)+\operatorname{Nil}(R / I)= \pm 1+I+\operatorname{Nil}(R / I)$. But if $z \in N i l(R / I)$, then $z=a+I$ with $a \in \operatorname{Nil}(R)$ because $I$ is nil. Therefore, one sees that $r \mp 1-a \in I \subseteq \operatorname{Nil}(R)$, which ensures that $r \in \pm 1+\operatorname{Nil}(R)$, as desired.

All of the above facts manifestly demonstrate that there is an abundance of WUU rings. So, it is rather natural to proceed with some of their special properties. The first point of the following statement supersedes the same fact for UU rings from [7].

Proposition 2.6. Let $R$ be a WUU ring. Then the following hold:

(1) $J(R)$ is a nil ideal.

(2) $3 \in U(R)$ if and only if $2 \in \operatorname{Nil}(R)$ and $2 \in U(R)$ if and only if $3 \in \operatorname{Nil}(R)$. In particular, if $3 \in \operatorname{Nil}(R)$, then $\operatorname{Id}(R)=\{0,1\}$.

Proof. (1) Let $x \in J(R)$ and suppose that $x \notin \operatorname{Nil}(R)$. Since $1+x \in U(R)=$ $\pm 1+\operatorname{Nil}(R)$, it must be that $1+x=-1+q$ for some $q \in \operatorname{Nil}(R)$, so that $2+x \in \operatorname{Nil}(R)$. Similarly, since $1+x^{2} \in U(R)$, we deduce that $x^{2}+2 \in \operatorname{Nil}(R)$. Hence it follows that their difference $\left(x^{2}+2\right)-(2+x)=x^{2}-x=-x(1-x) \in$ $\operatorname{Nil}(R)$. But $1-x \in U(R)$ whence $x \in \operatorname{Nil}(R)$, a contradiction. This substantiates our claim.

(2) Write $3=n-1$ or $3=n+1$ for some $n \in \operatorname{Nil}(R)$, which yields that $4=2^{2}=n$ or $2=n$. In both cases, we have $2 \in \operatorname{Nil}(R)$. Conversely, since $1+\operatorname{Nil}(R) \subseteq U(R)$, we have $1+2=3 \in U(R)$ whenever $2 \in \operatorname{Nil}(R)$.

Write $2=n-1$ or $2=n+1$, which implies that $3=n$ or $1=n$. Therefore, $3 \in \operatorname{Nil}(R)$. For the converse, since $1+\operatorname{Nil}(R) \subseteq U(R)$, we have $1+3=4=2^{2} \in$ $U(R)$, that is, $2 \in U(R)$ whenever $3 \in \operatorname{Nil}(R)$.

As for the second part, let $e$ be an idempotent in $R$. Clearly, $2 e-1$ is a unit, whence either $2 e-1=n-1$ or $2 e-1=n+1$. In the first case, $2 e=n$ and thus 
$e=\frac{n}{2}$ is a nilpotent which assures that $e=0$. In the second case, $2 e=n+2$ and so $e=\frac{n}{2}+1$ is a unit which ensures that $e=1$.

Note that the above proof illustrates that if $e$ is an arbitrary idempotent in a WUU ring, then either $2 e$ or $2(1-e)$ is always a nilpotent. Likewise, in a WUU ring, 6 cannot be a unit, because otherwise 2 and 3 will be both units which is against Proposition 2.6.

COROLlaRY 2.7. If $R$ is a WUU ring of characteristic $p^{t}$ for some prime $p$ and $t \in \mathbf{N}$, then $p=2$ or $p=3$.

Proof. If we assume that $p>3$, then it is plainly seen that $\left(2, p^{t}\right)=1$ and $\left(3, p^{t}\right)=1$, so that $2 \in U(R)$ and $3 \in U(R)$. But, furthermore, Proposition 2.6 applies to show that 2 and 3 are simultaneously nilpotents, which is a contradiction. This gives our assertion.

THeOREM 2.8. A ring $R$ is $U U$ if and only if $R$ is $W U U$ and $2 \in J(R)$.

Proof. The necessity follows immediately by [7, Theorem 2.6(1)].

As for the sufficiency, we process like this: First, if $2 \in \operatorname{Nil}(R)$, then we observe that WUU rings and UU rings do coincide since for any unit $u$ of $R$ we must have $u=n+1$ or $u=n-1$ for some nilpotent $n$. Thus, $u=n-1=$ $(n-2)+1$, where $n-2$ is a nilpotent, as required.

Furthermore, because $1+J(R) \leq U(R)$, it must be that $3 \in U(R)$ and so $3=n+1$ or $3=n-1$ for some nilpotent $n$. Therefore, $2=n$ or $4=2^{2}=n$. In both cases 2 is a nilpotent, and hence by what we have already shown above WUU and UU are equivalent conditions.

In [8, Proposition 1.12], and generally in [2], it was shown that if $R$ is a weakly nil-clean ring, then $6 \in \operatorname{Nil}(R)$. But whether or not this is true for WUU rings is not known yet. However, for clean rings, we offer the following:

Lemma 2.9. If $R$ is a clean $W U U$ ring, then $6 \in \operatorname{Nil}(R)$.

Proof. Write $3=e+u$, where $e \in I d(R)$ and $u \in U(R)$. We have two possibilities, that are, $u=1+q$ or $u=-1+q$, which being substituting give that $2=e+q$ or $4=e+q$ for some $q \in \operatorname{Nil}(R)$. Hence, it is easily verified 
that $e q=q e$ and therefore either $2=2^{2}-2=(e+q)^{2}-(e+q)=2 e q+q^{2}-q \epsilon$ $\operatorname{Nil}(R)$ or $12=4^{2}-4=(e+q)^{2}-(e+q)=2 e q+q^{2}-q \in \operatorname{Nil}(R)$. In the first case, it follows at once that $6=2.3 \in \operatorname{Nil}(R)$ or that $6^{2}=12.3 \in \operatorname{Nil}(R)$. So, in both cases, we get that $6 \in \operatorname{Nil}(R)$, as asserted.

A ring $R$ is called local if $R / J(R)$ is a division ring, that is, every its element lies in either $U(R)$ or in $J(R)$. Equivalently, $R$ is local exactly when it possesses a unique maximal left (respectively, right) ideal. In addition, if $R$ is commutative, then $R$ is local if and only if it has unique maximal ideal, say $J(R)$.

Note that any local ring is always a clean ring. In fact, $R$ is a local ring if and only if $R$ is an indecomposable clean ring, and hence an abelian clean ring. So, we derive the following consequence.

Proposition 2.10. Let $R$ be a local $W U U$ ring. Then $6 \in \operatorname{Nil}(R)$.

The following decomposition is essential.

THEOREM 2.11. Suppose that $R$ is a WUU ring for which $6 \in J(R)$. Then $R \cong R_{1} \times R_{2}$, where $R_{1}$ is a $U U$ ring and $R_{2}$ is either $\{0\}$ or is an indecomposable $W U U$ ring with $3 \in J\left(R_{2}\right)$.

Proof. Observe that for any $n \in \mathbf{N}$ we have $\left(2^{n}, 3^{n}\right)=1$, i.e., there exist non-zero integers $u, v$ such that $2^{n} u+3^{n} v=1$. This, consequently, allows us to write that $R=2^{n} R+3^{n} R$. Since $6 \in J(R)$, it follows that $5 \in U(R)$. Hence either $5=1+n$ or $5=-1+n$ with $n \in \operatorname{Nil}(R)$. Thus $4=2^{2}=n$, whence $2 \in \operatorname{Nil}(R)$, or $6=n \in \operatorname{Nil}(R)$. So, in both cases, 6 has to be a nilpotent element. This assures that $6^{m}=0$ for some $m \in \mathbf{N}$, and so $2^{m} R \cap 3^{m} R=\{0\}$; in fact, if $x=2^{m} a=3^{m} b$ for some $a, b \in R$, then $2^{m} a u=3^{m} b u$. However, $\left(1-3^{m} v\right) a=3^{m} b u$ whence $3^{m}(a v+b u)=a$. Multiplying both sides by $2^{m}$, we derive that $0=2^{m} a=x$, as needed.

Furthermore, we apply the classical Chinese Reminder Theorem to get that $R \cong\left(R / 2^{m} R\right) \times\left(R / 3^{m} R\right)$. So, we may put $R_{1}=R / 2^{m} R$ and $R_{2}=R / 3^{m} R$. This follows also directly, because $R=2^{m} R \oplus 3^{m} R$ and $R / 2^{m} R \cong 3^{m} R, R / 3^{m} R \cong 2^{m} R$.

Note that both $R_{1}$ and $R_{2}$ are WUU rings as special epimorphic images of $R$ being direct factors. Since $2 \in J\left(R_{1}\right)$, Theorem 2.8 gives that $R_{1}$ is a UU ring. On the other hand, since $3 \in J\left(R_{2}\right)$, it follows at once that $2 \in U\left(R_{2}\right)$ whence Proposition 2.6(2) ensures that $R_{2}$ is an indecomposable ring with $3 \in \operatorname{Nil}\left(R_{2}\right)$, as stated. 
Let $R$ be a ring. We recollect once again that, by [2, Theorem 12], $R$ is an abelian weakly nil-clean ring if and only if $J(R)$ is nil and $R / J(R)$ is isomorphic to either a boolean ring, or to $\mathbf{Z}_{3}$, or to the product of two such rings.

THeOREM 2.12. Suppose $R$ is a ring. Then $R$ is a clean WUU ring such that $2 \in U(R)$ if and only if $J(R)$ is nil and $R / J(R) \cong \mathbf{Z}_{3}$.

Proof. The sufficiency follows like this: Since -3 lies in $J(R)$ and $1+J(R) \subseteq U(R)$, it follows at once that 2 belongs to $U(R)$. Also, it follows by the aforementioned Theorem 12 from [2] that $R$ is weakly nil-clean. But it was established again in [2] that any weakly nil-clean ring is clean. Moreover,

$$
U(R) /(1+J(R)) \cong U(R / J(R)) \cong U\left(\mathbf{Z}_{3}\right)=\{-1,1\}
$$

and since $J(R) \subseteq \operatorname{Nil}(R)$, one deduces that $U(R)=\operatorname{Nil}(R) \pm 1$, as required.

As for the necessity, by Proposition 2.6(2), $R$ is indecomposable. Hence, each element $x$ is the form $u+1$ or $u$, where $u$ is a unit. If firstly $x=u+1$, then, because either $u=n+1$ or $u=n-1$, where $n$ is nilpotent, we obtain that $x=n+2$ or $x=n$. Since $2 \in U(R)$, clearly $n+2$ is also a unit, and hence $x=t+1$ or $x=t-1$, where $t$ is a nilpotent. Finally, $x$ is either the sum or the difference of a nilpotent and an idempotent. Secondly, if $x=u$, we deduce $x=q+1$ or $x=q-1$, where $q$ is a nilpotent. Hence $x$ has again the same property. Whence $R$ is an abelian weakly nil-clean ring and, in view of the listed above [2, Theorem 12], $J(R)$ is nil and $R / J(R)$ has to be isomorphic to $\mathbf{Z}_{3}$ because in conjunction with Proposition 2.6(2) the element 3 lies in $J(R)$ and so the factor-ring $R / J(R)$ is of characteristic 3 .

COROLlary 2.13. A local WUU ring $R$ is exactly a ring $R$ for which $J(R)$ is nil and $R / J(R)$ is isomorphic to either a boolean ring or to $\mathbf{Z}_{3}$.

Proof. It follows that either $2 \in U(R)$ or $2 \in J(R)$. In the first case, we just appeal to Theorem 2.12 to conclude the claim. In the second case, Theorem 2.8 applies to get that $R$ is a UU ring and hence [7, Theorem B] guarantees that $R / J(R)$ has to be boolean, as asserted.

As a valuable consequence, in parallel to Theorem 2.12, we yield its generalization (note that by Proposition 2.6(2) it must be that $3 \in \operatorname{Nil}(R)$ and hence $3 \in J(R)$ whenever $2 \in U(R))$ : 
COROLlaRY 2.14. Let $R$ be a ring. Then $R$ is a clean WUU ring if and only if $J(R)$ is nil and $R / J(R)$ is isomorphic to either a boolean ring $B$, or to $\mathbf{Z}_{3}$, or to $B \times \mathbf{Z}_{3}$.

Proof. Combining Lemma 2.9 and Theorem 2.11, we observe that the first factor $R_{1}$ must be a clean UU ring while the second factor $R_{2}$ must be an indecomposable clean WUU ring and hence a local WUU ring. We next just combine [7] with Corollary 2.13 to conclude the claim.

For the converse implication we can argue as in Proposition 2.3, seeing trivially that $R$ is WUU clean.

REMARK. The above Lemma 2.9 can now be successfully deduced directly from Corollary 2.14.

In [7], it was established that a clean UU ring is strongly nil-clean, and vice versa. We are now ready to improve that to the following:

COROLlary 2.15. The next four points are equivalent:

(i) $R$ is an exchange $W U U$ ring;

(ii) $R$ is a clean WUU ring;

(iii) $R$ is a weakly nil-clean ring having the strong property;

(iv) $J(R)$ is nil and $R / J(R)$ is isomorphic to either a boolean ring $B$, or to $\mathbf{Z}_{3}$, or to $B \times \mathbf{Z}_{3}$.

Proof. The equivalence (ii) $\Leftrightarrow$ (iv) follows from Corollary 2.14. The implication (iii) $\Rightarrow$ (ii) follows by [2, Corollary 8] and the fact that $R$ must be a WUU ring. In fact, given $u \in U(R)$, we have $u=n+e$ or $u=n-e$, where $n \in \operatorname{Nil}(R)$ and $e \in I d(R)$. Since $e n=n e$, we obtain that $u n=(n \pm e) n=$ $n^{2} \pm e n=n^{2} \pm n e=n(n \pm e)=n u$, so that $u-n$ is always a unit. Hence so is $\pm e$ and thus $e= \pm 1$. That is why $R$ is a WUU ring, as expected.

To show that (iv) $\Rightarrow$ (iii), it is a plain technical matter to check that $R=$ $J(R)+L \subseteq \operatorname{Nil}(R)+L$, where all elements of the set $L$ are central idempotents due to the fact that $R / J(R)$ is commutative, as required.

What remains to prove is that (i) $\Leftrightarrow$ (ii). The implication (ii) $\Rightarrow$ (i) being trivial, we deal with the (i) $\Rightarrow$ (ii) one. Utilizing Propositions 2.6(1) and 2.5, we may assume that $J(R)=0$. Furthermore, using the same idea as in [7], in view of Proposition 2.23, we conclude that $\operatorname{Nil}(R)=0$, so that $R$ has to be abelian. Hence $R$ is clean by virtue of [10], as needed. 
REMARK. For another verification of the equivalence (ii) $\Leftrightarrow$ (iii), we refer also to [6].

The following technicality is our crucial tool.

Lemma 2.16. Suppose $u$ is a unit and $e$ is an idempotent of a ring $R$ such that $u^{2} e=e u^{2}=e$ and $u=e+q$ or $u=-e+q$, where $q$ is a nilpotent. Then $e=1$.

Proof. Letting $u=e+q$, for some $e \in I d(R)$ and $q \in \operatorname{Nil}(R)$ with $q^{t}=0$, $t \in \mathbf{N}$ say, we obtain that $u^{2}=e+e q+q e+q^{2}$ and hence $u^{2} e=e=e+e q e+$ $q e+q^{2} e$ which forces that $\left(q+q^{2}\right) e=-e q e$, Similarly, $e u^{2}=e$ insures that $e\left(q+q^{2}\right)=-e q e$. Thus $e$ commutes with the nilpotent $\left(q+q^{2}\right)^{n}=[q(1+q)]^{n}=$ $q^{n}(1+q)^{n}$ for all $n \in \mathbf{N}$, and therefore the same is valid for $u$. Furthermore, $u-\left(q+q^{2}\right)=e-q^{2}$ with $u-\left(q+q^{2}\right)=u^{(2)}=e-q^{2}$ being a unit, one sees that $u^{(2)}-\left(2 q^{3}+q^{4}\right)=e-\left(q^{2}+2 q^{3}+q^{4}\right)=e-\left(q+q^{2}\right)^{2}$. Putting $u^{(3)}=u^{(2)}+$ $\left(q+q^{2}\right)^{2}$, we observe that $u^{(3)}$ is a unit since $u^{(2)}$ commutes with $\left(q+q^{2}\right)^{2}$ and that $u^{(3)}=e+q^{3}(2+q)$. Repeating the same procedure $t$-times, we will find a unit $u^{(t)}$ such that $u^{(t)}=e+q^{t} . a=e$ for some element $a \in R$ depending on $q$; actually $a=-1=-q^{0}$ when $t=2$. This yields that $e=1$, which exhausts this case.

Analogously, $\left(q^{2}-q\right) e=e\left(q^{2}-q\right)$ and $\left(q^{2}-q\right) u=u\left(q^{2}-q\right)$. Hence $u-q^{2}$ $=\left(q-q^{2}\right)-e$. The same trick as above successfully works to conclude the claim after all.

REMARK. This lemma improves an assertion from [11] which states that if $v$ is an involution, that is, $v^{2}=1$, with the property that $v=e+q$, where $e$ is an idempotent and $q$ is a nilpotent, then $e=1$, i.e., $v$ is a unipotent. However, his method is not applicable in the current situation because $u^{2}(1-e)=u^{2}-u^{2} e=$ $u^{2}-e=1-e$ provided a priori that $u^{2}=1$. But this cannot be deduced automatically, namely the fact that $u$ is an involution and so $u^{2}=1$ will follow after certain arguments.

We are now ready to describe completely arbitrary weakly nil-clean rings. Specifically, the following holds:

THeOREM 2.17. A ring $R$ is weakly nil-clean if and only if $R$ is either a nilclean ring, or $R / J(R)$ is isomorphic to $\mathbf{Z}_{3}$ with $J(R)$ nil, or $R$ is a direct product of two such rings. 
Proof. Firstly, let us assume that $R$ is weakly nil-clean. According to [2, Theorem 7], we consider only the case where 2 inverts in $R$. Letting $e$ be an arbitrary idempotent in $R$, we observe that $2 e-1$ is a weakly nil-clean involution in $R$ because $(2 e-1)^{2}=1$, so that with Lemma 2.16 at hand, applied to $u=2 e-1$, we deduce that either $2 e-1=1+q$ or $2 e-1=-1+q$ for some nilpotent $q$. In the first case, $2(1-e)$ is always a nilpotent whence so has to be $1-e$ which gives that $e=1$. In the second case, $2 e$ is a nilpotent and hence so is $e$ which implies that $e=0$. (Compare also with the note after the proof of Proposition 2.6.) Thus $R$ being indecomposable must be abelian and we next just employ [2, Theorem 12] to infer the claim.

Conversely, if the formulated conditions from the text of the theorem are satisfied, then we just apply a combination of [2, Theorem 2(3), Proposition 3] to get the wanted result.

REMARK. For another full description of weakly nil-clean rings the interested reader can see also [11].

Another nontrivial example of a WUU ring is the following one:

COROLlary 2.18. Any weakly nil-clean ring in which 2 inverts is WUU.

Proof. The proof of Theorem 2.17 enables us that such a ring has to be abelian, so that Proposition 2.3 applies to conclude the implication.

We shall say that a ring $R$ satisfies the nil-involution property if, for every $r \in R$, there exist a nilpotent $q$ and an involution $v$ such that $r=q+1+v$ or $r=q-1+v$.

The following result completely describes these rings.

Proposition 2.19. A ring has the nil-involution property if and only if it is weakly nil-clean with 2 invertible.

Proof. "Necessity". Let $a \in R$, where $R$ satisfies the nil-involution property. We first claim that $2 \in U(R)$. To prove that, we observe that the record $1=q+1+v$ is impossible, because otherwise $q=-v$, a contradiction. Thus $1=q-1+v$ whence $2=q+v$. Hence, multiplying by $v$ both sides from the left and from the right, respectively, we conclude that $v q=2 v-1=q v$. This yields at once that 2 is a unit, as claimed. 
Furthermore, one writes that $-2 a=u+v$ where $u \in \operatorname{Nil}(R) \pm 1$ and $v^{2}=1$. If $u=b+1$ with $b \in \operatorname{Nil}(R)$, then $a=(-b / 2)-(1+v) / 2$ with $-b / 2 \in \operatorname{Nil}(R)$ and $(1+v) / 2 \in \operatorname{Id}(R)$. In the remaining case, if $u=b-1$ with $b \in \operatorname{Nil}(R)$, then $a=(-b / 2)+(1-v) / 2$ with $-b / 2 \in \operatorname{Nil}(R)$ and $(1-v) / 2 \in \operatorname{Id}(R)$. So, $R$ is weakly nil-clean, indeed.

"Sufficiency". Suppose now $R$ is weakly nil-clean with $2 \in U(R)$. Note that in conjunction with [2] one has that $J(R)$ is nil and $3 \in J(R)$. Let $a \in R$. Then there exist $b \in \operatorname{Nil}(R)$ and $e^{2}=e \in R$ such that $a=b+e$ or $a=b-e$. If $a=b+e$, then $a=((b+3 e)-1)+(1-2 e)$ with $(1-2 e)^{2}=1$. Moreover, as $b^{m}=0$ for some $m>0$ and $3 \in J(R)$, we obtain that $(b+3 e)^{m} \in J(R)$, so $b+3 e$ is a nilpotent as $J(R)$ is nil. If $a=b-e$, then $a=((b-3 e)+1)+(-1+2 e)$ with $(-1+2 e)^{2}=1$. Moreover, as $b^{m}=0$ for some $m>0$ and $3 \in J(R)$, we deduce that $(b-3 e)^{m} \in J(R)$, so $b-3 e$ is a nilpotent as $J(R)$ is nil. Hence, $R$ satisfies the nil-involution property.

REMARK. In view of the last two assertions, we infer that all rings possessing the nil-involution property are WUU rings.

As an other concrete application of the results above, we shall completely characterize a proper subclass of the class of unit-regular rings: Recall that a ring $R$ is said to be unit-regular if for each $r \in R$ there exists $u \in U(R)$ such that $r=$ rur. It was established in [4] that each unit-regular ring is clean. In [5] we defined the so-called nil-regular rings as those rings $R$ having the property that, for every $r \in R$, there is $n \in \operatorname{Nil}(R)$ such that $r=r(1+n) r$. At first glance these are unit-regular UU rings. However, surprisingly, it was shown there that in such rings we must have $n=0$ and thus they are precisely the classical boolean rings in which any element is an idempotent.

Generalizing them, we call a ring $R$ weakly nil-regular if, for each $r \in R$, there exists $q \in \operatorname{Nil}(R)$ such that either $r=r(1+q) r$ or $r=r(-1+q) r$. Clearly, if $2 \in \operatorname{Nil}(R)$, then $-1+q=1+(q-2)$ with $q-2 \in \operatorname{Nil}(R)$, so that weakly nilregular rings will coincide with nil-regular rings.

So, we are now ready to proceed by proving the following:

Proposition 2.20. A ring $R$ is weakly nil-regular if and only if either $R \cong B$ or $R \cong \mathbf{Z}_{3}$ or $R \cong B \times \mathbf{Z}_{3}$, where $B$ is a boolean ring.

Proof. " $\Rightarrow "$. First of all, it is not hard to check that $J(R)=0$ because weakly nil-regular rings are themselves unit-regular and so regular rings. 
Next, given $u \in U(R)$, we either have $u^{-1}=u^{-1}(1+q) u^{-1}$ or $u^{-1}=$ $u^{-1}(-1+q) u^{-1}$. Hence $u=1+q$ or $u=-1+q$, so that $U(R)= \pm 1+\operatorname{Nil}(R)$ and thus $R$ is a WUU ring.

Moreover, one can write that $2=2(1+q) 2$ or that $2=2(-1+q) 2$. The first equality ensures that $2=4+4 q$, i.e., $2=-4 q \in \operatorname{Nil}(R)$, whereas the second one assures that $2=-4+4 q$, i.e., $6=4 q \in \operatorname{Nil}(R)$. Therefore, in both cases, it must be that $6 \in \operatorname{Nil}(R)$, whence $6 \in J(R)$ being a central nilpotent. Furthermore, since $R$ is obviously unit-regular, and hence clean, being the elements $\pm 1+q$ units, Corollary 2.14 applies to conclude the claim.

" $\Leftarrow$ ". One observes that for any idempotent $b$ the presentations $b=$ $b(1+0) b$ and $-b=(-b)(-1+0)(-b)$ hold, which guarantee our assertion.

We will now consider the element-wise behavior of clean elements in WUU rings. Specifically, the following is true:

THEOREM 2.21. In any abelian WUU ring $R$ with $6 \in \operatorname{Nil}(R)$, an element is clean if and only if it is weakly nil-clean.

Proof. By Proposition 2.5, working in the quotient $R / 6 R$, we without loss of generality may assume that $6=0$.

Suppose $R$ is a WUU ring and $a \in R$ is clean, saying that $a=u+e$, where $u \in U(R)$ and $e \in I d(R)$. Next, writing $u=q \pm 1$, where $q \in \operatorname{Nil}(R)$, it follows that $a=q \pm 1+e$ and so either $a=q+1+e$ or $a=q-1+e$. In the first case, $a=(2 e+q)+(1-e)$ while in the second one $a=q-(1-e)$. Since $1-e$ is an idempotent, it suffices to show that $2 e+q$ is a nilpotent. To that goal, as noted above, either $2 e$ or $2(1-e)$ is a nilpotent. In the first situation it follows at once that $2 e+q$ is again a nilpotent, and hence we are finished. In the latter one, we write $a=(2(e-1)+q)+(3-e)=(2(e-1)+q)-(3+e)$ bearing in mind that $-3=3$. Now, one sees that $2(e-1)+q$ is a nilpotent and $(3+e)^{2}=$ $9+6 e+e^{2}=9+7 e=3+e$ is an idempotent, so we are set.

Next, suppose that $R$ is a WUU ring and $r \in R$ is weakly nil-clean, setting that $r=q+e$ or $r=q-e$, where $q \in \operatorname{Nil}(R)$ and $e \in I d(R)$. In the second case, $r=(q-1)+(1-e)$, where $q-1$ is a unit and $1-e$ is an idempotent, so we are done. In the first case, $r=(q-1+2 e)+(1-e)$. Since $1-e$ is an idempotent, it needs to show that $q-1+2 e$ is a unit. If $2 e$ is a nilpotent, this is so because $q+2 e$ is a nilpotent, provided $2 e$ is a nilpotent. If now $2(1-e)$ is a nilpotent, we have that $r=(q-1+2(e-1))+(3-e)=(q-1+2(e-1))-(3+e)$ because 
$3=-3$. Since $q+2(e-1)$ is a nilpotent, whence $q-1+2(e-1)$ is a unit, and as above $3+e$ is a nilpotent, we conclude the wanted claim.

Since any local ring is indecomposable, and thus abelian, as a direct consequence, we derive the following:

COROLlary 2.22. In any local WUU ring, an element is clean if and only if it is weakly nil-clean.

Proof. It follows by a simple combination of Proposition 2.10 and Theorem 2.21 .

We will now deal with the corner and matrix questions for WUU rings.

Proposition 2.23. For any $e \in \operatorname{Id}(R)$, if the ring $R$ is $W U U$, then the corner ring eRe is also WUU.

Proof. Let $u \in U(e R e)$ with inverse $v \in U(e R e)$, that is $u v=v u=e$, whence $u+(1-e) \in U(R)$ with inverse $v+(1-e)$ because $u(1-e)=(1-e) u=v(1-e)$ $=(1-e) v=0$. Thus we can write $u+1-e=q+1$ or $u+1-e=q-1$ where $q \in \operatorname{Nil}(R)$. In the first case, $q=u-e \in e \operatorname{Re} \cap \operatorname{Nil}(R) \subseteq \operatorname{Nil}(e \operatorname{Re})$, so that $u=$ $q+e$ is a unipotent unit in $e$ Re.

In the second case, multiplying both sides of the equality $u+1-e=q-1$ by $e$ from the left and from the right, respectively, we deduce that $e u=e q-e$ and $u e=q e-e$. But it is easily seen that $e u=u e=u$ which guarantees that $e q=q e$ is a nilpotent. In addition, again multiplying the last equality by $e$, we obtain that $e q e=e q=q e$. Consequently, $u=e q-e$ is the difference of a nilpotent and identity in $e R e$. Finally, $u$ is a weak unipotent, as required.

As a valuable consequence, we derive:

COROLlaRY 2.24. For any nonzero ring $R$ and an arbitrary natural $n \geq 2$, the full matrix $n \times n$ ring $\mathbf{M}_{n}(R)$ is not a WUU ring.

Proof. Since $\mathbf{M}_{2}(R)$ is isomorphic to a corner ring of $\mathbf{M}_{n}(R)$, in conjunction with Proposition 2.23 it suffices to show that $\mathbf{M}_{2}(R)$ is not a WUU ring. So, consider the matrix unit $\left(\begin{array}{ll}0 & 1 \\ 1 & 1\end{array}\right)$ with inverse $\left(\begin{array}{cc}-1 & 1 \\ 1 & 0\end{array}\right)$. But $\left(\begin{array}{ll}0 & 1 \\ 1 & 1\end{array}\right)+$ 
$\left(\begin{array}{ll}1 & 0 \\ 0 & 1\end{array}\right)=\left(\begin{array}{ll}1 & 1 \\ 1 & 2\end{array}\right)$ cannot be a nilpotent, because it is a unit with inverse $\left(\begin{array}{cc}2 & -1 \\ -1 & 1\end{array}\right)$. Moreover, $\left(\begin{array}{ll}0 & 1 \\ 1 & 1\end{array}\right)-\left(\begin{array}{ll}1 & 0 \\ 0 & 1\end{array}\right)=\left(\begin{array}{cc}-1 & 1 \\ 1 & 0\end{array}\right)$ is also a non-nilpotent being a unit with inverse $\left(\begin{array}{ll}0 & 1 \\ 1 & 1\end{array}\right)$. We, consequently, conclude that $\mathbf{M}_{2}(R)$ is not WUU, indeed.

Proposition 2.25. Any subring of a WUU ring containing the same identity is also a WUU ring.

Proof. Suppose $S \leq R$. Letting $u \in U(S)$ we have $u \in U(R)$ and hence $u \in$ $1+\operatorname{Nil}(R)$ or $u \in-1+\operatorname{Nil}(R)$. Thus $u \pm 1 \in \operatorname{Nil}(R) \cap S \subseteq \operatorname{Nil}(S)$, as required.

The next assertion contrasts with the corresponding one from [7].

Proposition 2.26. Every finite direct product $R_{1} \times \cdots \times R_{n}$ with $n \in \mathbf{N}$ is a $W U U$ ring if and only if there exists an index $1 \leq j \leq n$ such that $R_{j}$ is aWUU ring and $R_{i}$ is a $U U$ ring for each $1 \leq i \neq j \leq n$.

Proof. Notice firstly that if $R=R_{1} \times \cdots \times R_{n}$, then it is readily checked that $U(R)=U\left(R_{1}\right) \times \cdots \times U\left(R_{n}\right)$.

"Necessity". Since all $R_{i} \leq R, i \in[1, n]$, an appeal to Proposition 2.25 gives that these $R_{i}$ are WUU rings. Since $U(R)= \pm 1+N i l(R)$, that is, $U\left(R_{1}\right) \times \cdots \times$ $U\left(R_{n}\right)= \pm(1, \ldots, 1)+\operatorname{Nil}\left(R_{1}\right) \times \cdots \times \operatorname{Nil}\left(R_{n}\right)$, by considering units of the kind $(-1, \ldots, 1, \ldots-1),(1, \ldots,-1, \ldots 1)$ etc., we detect that $2 \in \operatorname{Nil}\left(R_{i}\right)$ for almost all indices $i \in[1, n]$ with the exception of exact one index $j \in[1, n]$. So, according to Theorem 2.8, these $R_{i}$ have to be UU rings, as promised.

"Sufficiency". We have $U\left(R_{j}\right)= \pm 1+\operatorname{Nil}\left(R_{j}\right)$ for some $1 \leq j \leq n$, and for all other $i \neq j$ from $[1, n]$ we have $U\left(R_{i}\right)=1+\operatorname{Nil}\left(R_{i}\right)$. Furthermore, if $U\left(R_{j}\right)=$ $1+\operatorname{Nil}\left(R_{j}\right)$, then $U(R)=1+\operatorname{Nil}(R)$ and so we are set. Otherwise, if $U\left(R_{j}\right)=$ $-1+\operatorname{Nil}\left(R_{j}\right)$, then we may also write $U\left(R_{i}\right)=-1+\operatorname{Nil}\left(R_{i}\right)$ for all $i \neq j$ and hence $U(R)=-1+\operatorname{Nil}(R)$, as needed.

As an immediate consequence, we yield:

COROLlaRy 2.27. For any $n \geq 2$ the ring $R^{n}$ is $W U U$ if and only if $R^{n}$ is $U U$ if and only if $R$ is $U U$. 
Proof. The first equivalence follows directly from Proposition 2.26. The second one follows from [7].

Standardly, $\mathbf{T}_{n}(R)$ will denote the ring of $n \times n$ upper triangular matrices over the ring $R$, where $n \geq 1$ is an integer.

As a consequence, we deduce:

COROllaRY 2.28. For any $n \geq 2$ the ring $\mathbf{T}_{n}(R)$ is a WUU ring if and only if $R$ is a UU ring.

Proof. Consider the set $I=\left\{\left(a_{i j}\right) \in \mathbf{T}_{n}(R): \forall a_{i i}=0\right\}$. It is not too hard to verify that $I$ is a nil ideal of $\mathbf{T}_{n}(R)$ such that $\mathbf{T}_{n}(R) / I \cong R^{n}$. We next apply Proposition 2.5 and Corollary 2.27 to get the wanted assertion.

Remark. In conjunction with [7], it follows the surprising fact that $\mathbf{T}_{n}(R)$ is WUU if and only if $\mathbf{T}_{n}(R)$ is UU. Moreover, owing to Corollary 2.28, the ring $\mathbf{T}_{n}\left(\mathbf{Z}_{3}\right)$ is not WUU, because in accordance with [7] the ring $\mathbf{Z}_{3}$ is not UU.

We shall now give a more direct and a slightly simpler proof of Theorem A from [7] in the case of characteristic 2 .

Proposition 2.29. A ring $R$ of $\operatorname{char}(R)=2$ is a $U U$ ring if and only if $U(R)$ is a 2-group.

Proof. " $\Rightarrow "$. Given $u \in U(R)$, it follows that $u=1+d$, where $d \in \operatorname{Nil}(R)$ with $d^{k}=0$ for some $k \in \mathbf{N}$. Hence $d^{2^{s}}=0$ for some sufficiently large natural $2^{s} \geq k$ and the Frobenius' law implies that $u^{2^{s}}=1$, as required.

" $\Leftarrow$ ". If $v \in U(R)$, then $v^{2^{i}}=1$ for some positive integer $i$ and, therefore, by the Frobenius' law we have $(v-1)^{2^{i}}=0$. Thus $v-1 \in \operatorname{Nil}(R)$ and $v \in 1+\operatorname{Nil}(R)$, as needed to get that $U(R) \subseteq 1+\operatorname{Nil}(R)$. The reverse inclusion is obvious.

Remark. Since in UU rings we have $-1=1+q$ for some $q \in \operatorname{Nil}(R)$, we obtain that $2 \in \operatorname{Nil}(R)$. So, $\operatorname{char}(R)=2^{k}$ for some $k \in \mathbf{N}$. So, in the proof of [7, Theorem 2.6(1)] the fact that $2^{t}=0$ for some $t \geq 1$ follows directly from the containment $2 \in \operatorname{Nil}(R)$. 
Proposition 2.30. If $R$ is a WUU ring of characteristic $p$ for some prime, then $U(R)$ is a 2p-group, especially a 2-group or a 6-group.

Proof. For any $u \in U(R)$, we can write either $u=q+1$ or $u=q-1$, where $q$ is a nilpotent. It must be that $u^{p^{j}}=1$ or $u^{p^{j}}=-1$ for some $j \in \mathbf{N}$. If both cases we have $u^{2 p^{j}}=u^{(2 p)^{j}}=1$, as required. The final part follows at once by applying Corollary 2.7 .

We now possess all the ingredients to prove the following assertion.

THEOREM 2.31. Suppose $R$ is a WUU ring of characteristic 6. Then $U(R)$ is a 6-group.

Proof. By virtue of Theorem 2.11, we write that $U(R) \cong U\left(R_{1}\right) \times U\left(R_{2}\right)$, where $R_{1}$ is a UU ring and $R_{2}$ is an indecomposable WUU ring with $3 \in J\left(R_{2}\right)$. Since $\operatorname{char}\left(R_{1}\right)=2$, in view of Proposition 2.29, $U\left(R_{1}\right)$ is a 2-group. Since in $R_{2}$ we must have $6=0$ and $2^{2}=1+3 \in U\left(R_{2}\right)$, i.e., $6=0$ and $2 \in U\left(R_{2}\right)$, we deduce that $3=0$ and $\operatorname{char}\left(R_{2}\right)=3$. We now apply Proposition 2.30 to get that $U\left(R_{2}\right)$ is a 6-group. Finally, we conclude that $U(R)$ is also a 6-group, as stated.

As usual, $R[X]$ denotes the polynomial ring over an arbitrary ring $R$. The following extends Corollary 2.2 from [3] and somewhat clarifies its proof.

THEOREM 2.32. Suppose $R$ is a commutative ring. Then $R[X]$ is a WUU ring if and only if $R$ is a WUU ring.

Proof. The necessity follows at once by Proposition 2.25 taking into account that $R$ is a subring of $R[X]$.

As to begin with the sufficiency, recall the following basic facts: Let $f=$ $a_{0}+a_{1} X+\cdots+a_{n} X^{n} \in R[X]$ be a polynomial with $n \in \mathbf{N}$. Then $f$ is a unit in $R[X]$ if and only if $a_{0}$ is a unit in $R$ and $a_{1}, a_{2}, \ldots, a_{n} \in N(R)$ are nilpotents in $R$ as well as $f$ is a nilpotent of $R[X]$ if and only if all the coefficients $a_{0}, a_{1}, a_{2}, \ldots, a_{n}$ are nilpotents of $R$. Furthermore, for any invertible polynomial $g$, we have that $g=b_{0}+b_{1} X+\cdots+b_{n} X^{n}=\left( \pm 1+a_{0}\right)+b_{1} X+\cdots+b_{n} X^{n}$ $= \pm 1+\left(a_{0}+b_{1} X+\cdots+b_{n} X^{n}\right)$, where $a_{0}$ is a nilpotent. Thus, it follows immediately from the stated above facts that $a_{0}+b_{1} X+\cdots+b_{n} X^{n}$ is a nilpotent polynomial, as required. 


\section{Open Questions}

In closing, we pose the following unanswered problems of interest:

Problem 1. Describe the group $U(R)$ of a WUU ring $R$.

A reasonable question is whether or not it is a 6-group, and eventually the ring $R$ is of characteristic 6, provided that $R$ is not UU of positive characteristic. However, it looks like there exist some "unexpected" examples of WUU rings with a strange group of units. For example, take the ring consisting of all matrices $\left(\begin{array}{ll}a & b \\ 0 & a\end{array}\right)$, where $a, b \in \mathbf{Z}$. The unit group of this ring has infinite order, although it is a subring of $\mathbf{T}_{2}(\mathbf{Z})$.

Problem 2. Does it follow that a WUU ring $R$ which is not UU can be decomposed as $R=R_{1} \times R_{2}$, where $R_{1}$ is a UU ring and $R_{2}$ is a local WUU ring?

Problem 3. For a ring $R$ describe when the rings $R(X)$ and $R\langle X\rangle$ are UU rings, respectively WUU rings.

Problem 4. Describe when group rings are UU rings, respectively WUU rings.

Problem 5. If $R$ is a WUU ring, does it follow that $R / J(R)$ is abelian?

Problem 6. Describe all UI rings that are rings in which each involution is a unipotent.

Notice that in [11] it was established that any nil-clean involution is a unipotent and thus each nil-clean ring is an UI ring. This extends Diesl's result that every strongly nil-clean unit is a unipotent and thereby each strongly nilclean ring is a UU ring.

Problem 7. Describe all WUI rings that are rings in which every involution is either a unipotent or minus a unipotent, i.e., is a weak unipotent.

More generally, we demonstrated in Lemma 2.16 that weakly nil-clean involutions are weak unipotents and so weakly nil-clean rings are WUI rings. 
An element $w$ is called $a$ weak involution if either $w^{2}=1$ or $w^{2}=-1$. So, we come to the final

Problem 8. Describe exchange rings with weak involutions.

\section{Acknowledgements}

The author owes his sincere thanks to Dr. J. Šter and Prof. T. Y. Lam for their valuable correspondence on the present topic. He is also deeply appreciated to the expert referees for their competent and insightful suggestions made.

\section{References}

[1] F. W. Anderson and K. R. Fuller, Rings and Categories of Modules, Springer-Verlag, BelinHeidelberg-New York, 1974.

[2] S. Breaz, P. Danchev and Y. Zhou, Rings in which every element is either a sum or a difference of a nilpotent and an idempotent, J. Algebra Appl. 15 (2016).

[ 3 ] G. Călugăreanu, UU rings, Carpathian J. Math. 31 (2015), 157-163.

[4] V. P. Camillo and D. Khurana, A characterization of unit regular rings, Commun. Algebra 29 (2001), 2293-2295.

[5] P. V. Danchev, A new characterization of boolean rings with identity, Irish Math. Soc. Bull. 76 (2015), 55-60.

[6] P. V. Danchev, A class of weakly nil-clean rings, Irish Math. Soc. Bull. 77 (2016), 9-17.

[7] P. V. Danchev and T. Y. Lam, Rings with unipotent units, Publicat. Math. (Debrecen) 88 (2016), 449-466.

[ 8 ] P. V. Danchev and W. Wm. McGovern, Commutative weakly nil clean unital rings, J. Algebra 425 (2015), 410-422.

[9] T. Y. Lam, A First Course in Noncommutative Rings, Second Edition, Graduate Texts in Math., Vol. 131, Springer-Verlag, Berlin-Heidelberg-New York, 2001.

[10] W. K. Nicholson, Lifting idempotents and exchange rings, Trans. Amer. Math. Soc. 229 (1977), 269-278.

[11] J. Šter, Nil clean involutions, arXiv preprint, 2015.

Department of Mathematics

Plovdiv University

4000 Plovdiv, Bulgaria

E-mail: pvdanchev@yahoo.com; peter.danchev@yahoo.com 